\title{
Photothermal Mid-Infrared Spectroscopy using Fano Resonances in Silicon Microring Resonators
}

\author{
A. Vasiliev, A. Malik, M. Muneeb, R. Baets and G. Roelkens \\ Photonics Research Group, INTEC-department, Ghent University-imec; Center for Nano- and BioPhotonics, Ghent \\ University, Sint-Pietersnieuwstraat 41, 9000 Ghent, Belgium \\ anton.vasiliev@ugent.be
}

\begin{abstract}
A novel photothermal mid-infrared spectroscopy method is demonstrated in the $3.5 \mu m$ wavelength range on a polymer analyte using an asymmetric Fano-type resonance in a silicon-on-insulator microring resonator.
\end{abstract}

OCIS codes: (130.0130) Integrated optics devices, (300.6430) Spectroscopy, photothermal

\section{Introduction}

Spectroscopy in the mid-infrared wavelength range is attractive for many sensing applications because of the high absorption cross-section of molecules and the increased selectivity through the molecule-specific 'fingerprint' absorption spectrum. Miniaturization of these optical devices presents great challenges due to the reduced interaction path length with the analyte. Furthermore, conventional mid-infrared absorption spectroscopy methods require an expensive and bulky cooled detector.

In recent years, examples of indirect measurement techniques such as photothermal- and photo-acoustic spectroscopy have promised to result in compact systems and have comparable if not better performance compared to classical absorption spectroscopy systems. In such systems the absorption of the pump beam induces a change of the thermodynamic parameters: pressure, density and temperature.

The combination of mid-infrared photothermal spectroscopy with resonant structures on a Photonic Integrated Chip (PIC) is paving the way for new highly selective and sensitive sensors $[1,2]$. In this work, a novel photothermal spectroscopic sensor is demonstrated which uses a sharp Fano-type resonance on a Silicon-On-Insulator (SOI) chip to transduce the temperature change of a polymer analyte, induced by optical absorption in the mid-infrared wavelength range (3250-3650 $\mathrm{nm}$ or $\left.3075-2740 \mathrm{~cm}^{-1}\right)$. The probing mechanism is explained in the caption of Figure 1 (a).

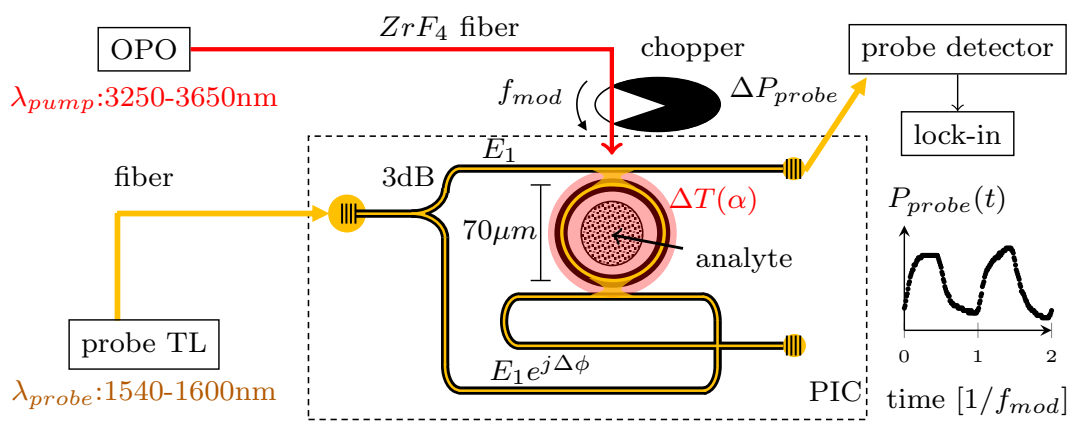

(a)

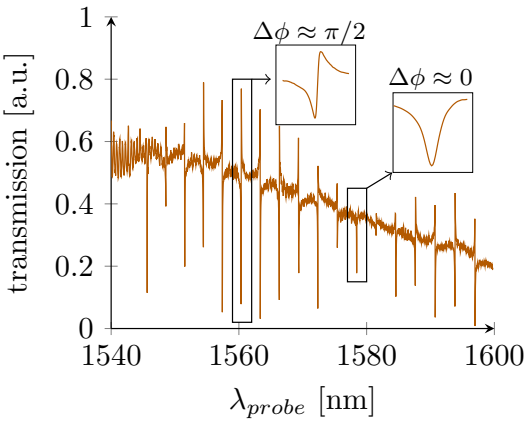

(b)

Fig. 1: Schematic of the photothermal spectroscopy experiment. (a) A fiber-coupled Optical Parametric Oscillator (OPO) illuminates the patterned analyte on the microring from $\approx 100 \mu \mathrm{m}$ distance. $\lambda_{\text {pump }}$ is scanned across the mid-infrared absorption spectrum of the analyte $\alpha(\lambda)$. Optical absorption leads to wavelength selective heat generation $\Delta T(\alpha)$ which is transduced to a change of effective index through the thermo-optic effect. This changes the resonance wavelength of the ring and thus the probe transmission $P_{\text {probe }}$ for a given $\lambda_{\text {probe }}$. The probe modulation is read out using a lock-in amplifier at the modulation frequency $f_{\text {mod }}$. (b) The probe laser (TL) scans the PIC and sets $\lambda_{\text {probe }}$ where the transmission slope is maximal. 


\section{Experiments}

A photoresist (AZ9260 from MicroChem), acting as a polymer analyte, with a thickness of $2 \mu m$ is lithographically patterned inside the annular region of a microring resonator on the PIC in Figure 1(a). Input probe light in the telecom wavelength range is coupled using standard grating couplers to the circuit. Light is split into two arms which are then coupled co-directionally into the ring, creating a response that depends on the phase difference $\Delta \phi$ between both ports [3]. There is no constraint on the exact value for $\lambda_{\text {probe }}$ as long as it is inside the probe laser range and within the grating coupler bandwidth. A suitable sharp resonance can therefore be found without active tuning of the device.

The lower arm is chosen to be $8 \mu \mathrm{m}$ longer such that the phase difference $\Delta \phi$ varies approximately linear with $\lambda_{\text {probe }}: \Delta \phi / \Delta \lambda \approx \pi / 35 \mathrm{~nm}$. With a ring radius of $35 \mu \mathrm{m}$ (free spectral range of $\approx 3.1 \mathrm{~nm}$ ), this results in a sampling of about 10 resonances between $\Delta \phi=0$ and $\Delta \phi=\pi$. When $\Delta \phi=m_{o d d} \times \pi / 2$ the transmission has the desired Fano-type lineshape with higher slope than when the two arms are in phase $\Delta \phi \approx 0$ resulting in a stronger signal, as also seen in Figures 2 (a) and (b).

The mid-infrared source is step-scanned with $5 \mathrm{~nm}$ resolution and for each pump wavelength $\lambda_{\text {pump }}$, the probe laser is scanned across the sharp Fano resonance and the maximal probe modulation is recorded. The effective temperature modulation of the microring is of the order of a few $\mathrm{mK}$ and is estimated by normalizing the photothermal response to the slope of the transmission $d \mathbb{T} / d \lambda, P_{\text {probe }}, \lambda_{\text {res }}$ and the thermo-optic coefficient $d n / d T$.

The obtained photothermal spectrum matches well with the one obtained using conventional Fourier-Transform InfraRed (FTIR) spectroscopy, see Figure 2 (c). The absorption peaks in this wavelength range correspond to the $\mathrm{C}-\mathrm{H}$-stretch vibrational frequencies of the polymer analyte.

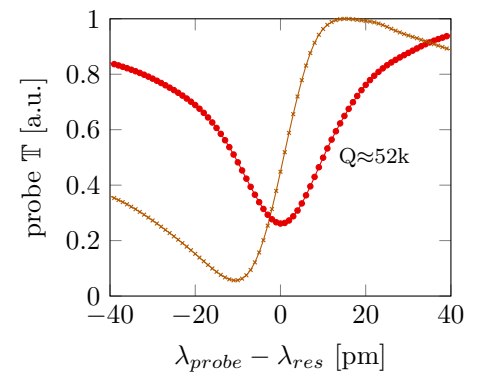

(a)

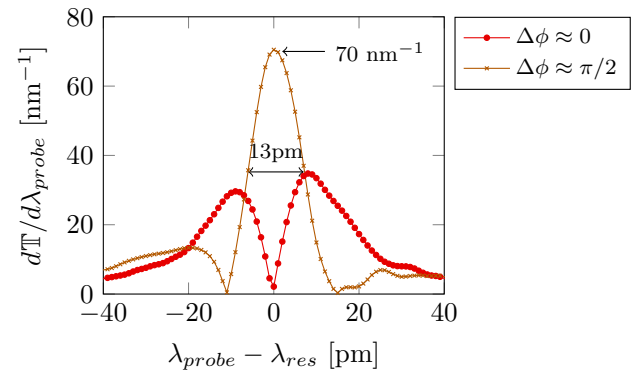

(b)

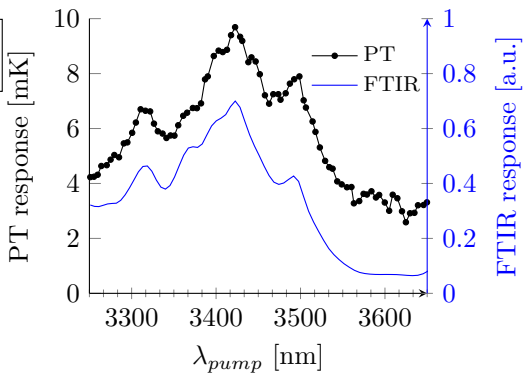

(c)

Fig. 2: (a) Measured near-infrared transmission spectrum near resonance of the Fano circuit showing the asymmetric Fano type lineshape when the phase difference between the two ports is $\Delta \phi \approx m_{\text {odd }} \times \pi / 2$ and compared to when $\Delta \phi \approx 0$. (b) The transmission slope $d \mathbb{T} / d \lambda$ is estimated from (a). (c) The probe modulation depth $\Delta P_{\text {probe }}$ is proportional to the absorption of the analyte at the mid-infrared wavelength $\lambda_{\text {pump }}$ and the slope $d \mathbb{T} / d \lambda$. The maximal probe modulation depth at each $\lambda_{\text {pump }}$ is recorded as the OPO is swept across the absorption spectrum. The recovered photothermal (PT) spectrum is normalized in such a way to estimate the effective temperature modulation at each point. The result corresponds well with the absorption spectrum obtained by classical FTIR spectroscopy.

\section{Conclusion}

A novel photothermal mid-infrared spectroscopy method is demonstrated in the $3.5 \mu \mathrm{m}$ wavelength range on a polymer analyte using an asymmetric Fano-type resonance in a silicon-on-insulator microring resonator. The polymer absorption spectrum was transduced photo-thermally, agreeing well with the spectrum retrieved through a bench-mark FTIR system. This result paves the way to realizing a mid-infrared photothermal spectroscopy gas sensor, by applying a gas adsorbing coating instead of the polymer layer [4].

\section{References}

[1] Juejun $\mathrm{Hu}$, et al. "Photothermal nano-cavities for ultra-sensitive chem-bio detection," Proc. SPIE 8018, Sensing XII, 80180W (2011)

[2] Kevin D. Heylman, et al. "Photothermal Microscopy of Nonluminescent Single Particles Enabled by Optical Microresonators," J. Phys. Chem. Lett. 5(11), 1917-1923 (2014).

[3] Ting Hu, et al. "Tunable Fano resonances based on two-beam interference in microring resonator," Appl. Phys. Lett. 102, 011112 (2013).

[4] Nebiyu A. Yebo, et al. "Selective and reversible ammonia gas detection with nanoporous film functionalized silicon photonic micro-ring resonator," Optics Express 20, 11855-11862 (2012). 